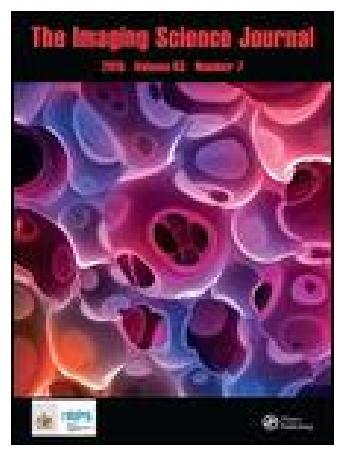

The Imaging Science Journal

\title{
A comparative study of light field representation and integral imaging
}

\section{E Sahin \& L Onural}

To cite this article: E Sahin \& L Onural (2010) A comparative study of light field representation and integral imaging, The Imaging Science Journal, 58:1, 28-31, DOI: 10.1179/136821909X12581187859817

To link to this article: http://dx.doi.org/10.1179/136821909X12581187859817

\section{曲 Published online: 18 Jul 2013.}

Submit your article to this journal $\pi$

Џll Article views: 29

Q View related articles $\sqsubset$

Citing articles: 3 View citing articles 4 


\title{
A comparative study of light field representation and integral imaging
}

\author{
E Sahin* and L Onural \\ Department of Electrical and Electronics Engineering, Bilkent University, Ankara TR-06800, Turkey
}

\begin{abstract}
Light field representation is a model for three-dimensional (3D) image representation and integral imaging is an optical 3D imaging and representation method. A comparative investigation of light field representation and integral imaging is given in this paper. The practical integral imaging is shown to be equivalent to the discrete light field representation if some restrictions are imposed on the light field. On the other hand, it is shown that the integral imaging is not equivalent to the continuous light field representation. In any case, physical realisation of an arbitrary abstract light field representation may not be possible due to restrictions associated with the uncertainty principle related to the spatial and angular resolutions.
\end{abstract}

Keywords: light field representation, integral imaging

\section{INTRODUCTION}

The appearance of the world at any time from any given point through any given direction can be represented with the so called plenoptic (plenty of optic) function, $P(x, y, z, \theta, \phi, \lambda, t){ }^{1}$ In other words, the plenoptic function corresponds to the radiance associated with the light ray, at time $t$, with wavelength $\lambda$, which passes from a three-dimensional (3D) point $(x, y, z)$ towards the direction $(\theta, \phi)$. In order to ease the notation, we will omit the variation with respect to $t$ and $\lambda$. With further assumption of free space propagation and by keeping the viewing positions outside the convex hull of the scene, one can also reduce the dimension of the plenoptic function to four since the radiance associated with the light ray along its path will be constant in this case. ${ }^{2}$

\section{LIGHT FIELD REPRESENTATION}

The four-dimensional plenoptic function is called light field. ${ }^{2}$ The light field is an abstract representation of

The MS was accepted for publication on 3 September 2009.

* Corresponding author: E Sahin, Department of Electrical and Electronics Engineering, Bilkent University, Ankara TR-06800, Turkey; email: sahin@ee.bilkent.edu.tr the optical power flow associated with the light rays. Let us define the infinitesimal power emanating from differential surface $\mathrm{d} A_{1}$ (on an arbitrary surface $W_{1}$ ) and reaching to the differential surface $\mathrm{d} A_{2}$ (on another arbitrary surface $W_{2}$ ) as $\mathrm{d} P$ (Fig. 1). We then associate a power density (for the power flow between $W_{1}$ and $W_{2}$ surfaces) to the ray crossing the two surfaces at $\left(x_{1}, y_{1}\right)$ and $\left(x_{2}, y_{2}\right)$ and define it as

$$
L\left(x_{1}, y_{1}, x_{2}, y_{2}\right)=\frac{\mathrm{d} P}{\mathrm{~d} A_{1} \mathrm{~d} A_{2}}
$$

We call $L\left(x_{1}, y_{1}, x_{2}, y_{2}\right)$ as the light field which can also be called ray power density by taking into account physical quantities into consideration.

Instead of using the second surface and the differential area $\mathrm{d} A_{2}$ on it, it is quite common to adopt a solid angle model in the literature, ${ }^{1,3}$ and the density in that case is called the radiance. However, we prefer the definition as given by equation (1) for our purposes. Incidentally, it is straightforward to establish the relation between the density given in equation (1) and the radiance by first noting that the differential solid angle is related to the differential area $\mathrm{d} A_{2}$ as $\mathrm{d} \Omega=\mathrm{d} A_{2} \cos \alpha_{2} / D^{2}$, where $\alpha_{2}$ is the angle between the ray and the outward normal to the $W_{2}$ surface at $\left(x_{2}, y_{2}\right)$ and $D$ is the distance between $\left(x_{1}, y_{1}\right)$ 


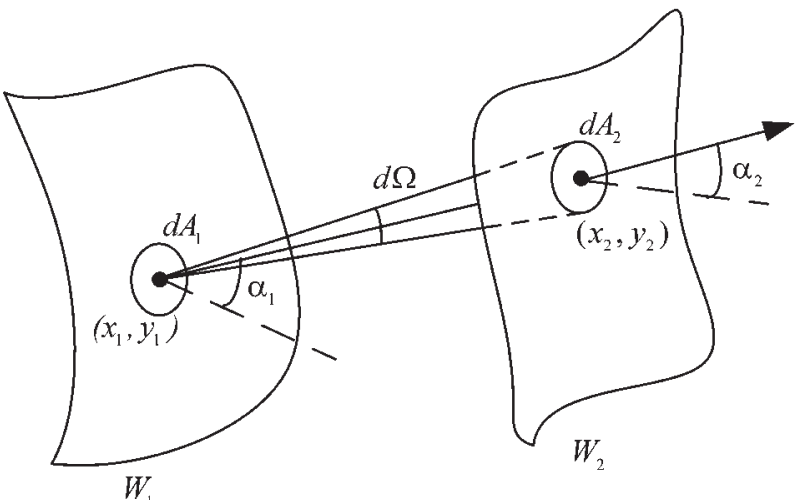

1 The relation of solid angle $\mathrm{d} \Omega$ with the differential area $\mathrm{d} A_{2}$

and $\left(x_{2}, y_{2}\right)$ (Fig. 1). And then the radiance associated with the ray crossing the two surfaces at $\left(x_{1}, y_{1}\right)$ and $\left(x_{2}, y_{2}\right)$ becomes

$$
\widetilde{L}\left(x_{1}, y_{1}, x_{2}, y_{2}\right)=\frac{\mathrm{d} P}{\mathrm{~d} A_{1} \cos \alpha_{1} \mathrm{~d} \Omega}
$$

where $\mathrm{d} P$ is the radiant power emanating from $\mathrm{d} A_{1}$ and propagating along the cone represented by the solid angle $\mathrm{d} \Omega, \mathrm{d} A_{1} \cos \alpha_{1}$ is the projected differential area on $W_{1}$ along the direction of the ray and $\mathrm{d} \Omega$ is the solid angle subtended by $A_{2}$. Therefore, $L$ and $\widetilde{L}$ are closely related to each other with a normalisation as

$$
L\left(x_{1}, y_{1}, x_{2}, y_{2}\right)=\frac{\tilde{L}\left(x_{1}, y_{1}, x_{2}, y_{2}\right)}{D^{2}} \cos \alpha_{1} \cos \alpha_{2}
$$

Discretisation of $L\left(x_{1}, y_{1}, x_{2}, y_{2}\right)$ is necessary for digital processing. Instead of arbitrary surfaces, we assume the simple two-parallel plane model ${ }^{2,4}$ where the $P_{1}$ and $P_{2}$ planes are usually defined as the camera and image planes at $z=z_{1}$ and $z=z_{2}$, respectively. Let us assume that the index arrays $\left[m_{1}, n_{1}\right]$ and $\left[m_{2}, n_{2}\right]$ represent the locations $\left(m_{1} M_{1}, n_{1} N_{1}, z_{1}\right)$ and $\left(m_{2} M_{2}, n_{2} N_{2}, z_{2}\right)$ on the two parallel planes, respectively, where $M_{1}, N_{1}, M_{2}$ and $N_{2}$ are the sampling intervals. Therefore, $\left[m_{1}, n_{1}\right]$ represents the centres of cameras (ideal pinhole camera model), and $\left[m_{2}, n_{2}\right]$ represents the sample points of the images that are taken by the cameras. We define the discrete power density of the ray crossing the $P_{1}$ and $P_{2}$ planes, following the definition given by equation (1), as

$$
L_{\mathrm{d}}\left[m_{1}, n_{1}, m_{2}, n_{2}\right]=\frac{P\left[m_{1}, n_{1}, m_{2}, n_{2}\right]}{S_{1} S_{2}}
$$

where $S_{1}$ and $S_{2}$ are the areas of the pixels on the $P_{1}$ and $P_{2}$ planes, respectively, $P\left[m_{1}, n_{1}, m_{2}, n_{2}\right]$ is the power emanating from the pixel represented by

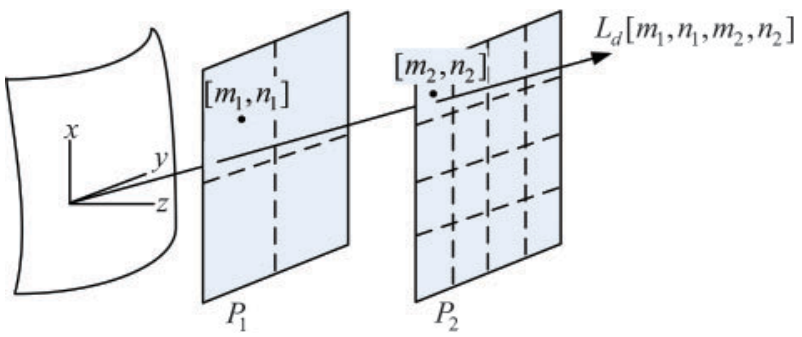

2 Representation of the ray power density in the discrete case

$\left[m_{1}, n_{1}\right]$ and reaching to the pixel represented by $\left[m_{2}, n_{2}\right]$ (Fig. 2). Therefore, $L_{\mathrm{d}}$ represents the power flow between the two pixels: $\left[m_{1}, n_{1}\right]$ on $P_{1}$ and $\left[m_{2}, n_{2}\right]$ on $P_{2}$. The subscript $d$ denotes that the field is discrete.

\section{INTEGRAL IMAGING}

Integral imaging is a $3 \mathrm{D}$ imaging method. It provides autostereoscopic images (allows 3D viewing without wearing glasses) of $3 \mathrm{D}$ scenes. ${ }^{5}$ The image is captured on a two-dimensional sensor array by a two-dimensional microlens array where the sensor array is placed behind the microlens array in a parallel fashion. Each microlens takes its own image of the $3 \mathrm{D}$ scene. The image that is formed behind each microlens is called elemental image. Therefore, the parameterisation of the integral imaging is the same as the two-plane parameterisation of the light field representation. $P_{1}$ is the plane on which the microlens array is placed and $P_{2}$ is the plane of the twodimensional sensor array.

The display stage of integral imaging is constructed by placing the same microlens array used at the imaging stage in front of the two-dimensional display device displaying the elemental images captured by the two-dimensional sensor array. The integral imaging renders a pseudoscopic $3 \mathrm{D}$ reconstruction to the observer. There are several ways to convert the pseudoscopic images to orthoscopic ones. ${ }^{6}$ Here in this paper, we consider only the recording stage. It is trivial to include the display phase in the discussion.

\section{RELATION OF LIGHT FIELD REPRESENTATION TO INTEGRAL IMAGING}

The counterpart of two parallel planes of light field representation in integral imaging is the microlens 


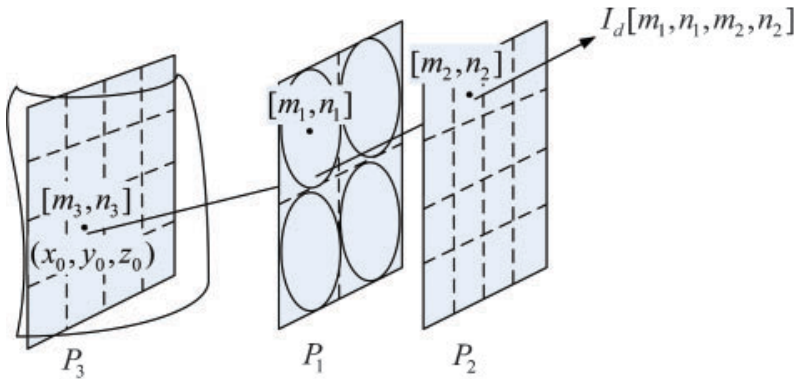

3 Parameterisation of the light power density in integral imaging

array plane $P_{1}$ and the sensor array plane $P_{2}$. However, the $P_{2}$ plane can be easily replaced by its image $P_{3}$ which is a hypothetical plane intersecting the $3 \mathrm{D}$ object volume (Fig. 3). It is assumed that the captured elemental images are all in focus; by the way, this is necessary for a successful result in integral imaging. We will include both $\left(P_{1}, P_{2}\right)$ and $\left(P_{1}, P_{3}\right)$ plane pairs in the discussion.

Let the light field between $P_{3}$ and $P_{1}$ planes be parameterized via the discrete light field representation $\hat{L}_{\mathrm{d}}\left[m_{3}, n_{3}, m_{1}, n_{1}\right]$. Let the $\left[m_{1}, n_{1}\right]$ array represent the locations of the microlenses where the aperture of each microlens corresponds to a pixel on the $P_{1}$ plane. Following the definition given by equation (4), one can find the power density of the light emanating from $\left(x_{0}, y_{0}, z_{0}\right)$ on the object surface and crossing the $P_{3}$ plane at $\left[m_{3}, n_{3}\right]$ and reaching the microlens at $\left[m_{1}, n_{1}\right]$ as

$$
\hat{I}_{\mathrm{d}}\left[m_{3}, n_{3}, m_{1}, n_{1}\right]=\frac{P\left[m_{3}, n_{3}, m_{1}, n_{1}\right]}{S_{1} S_{3}}
$$

where $S_{3}$ is the area of a pixel on $P_{3}, S_{1}$ is the area of the aperture of the microlens on $P_{1}$ and $P\left[m_{3}, n_{3}, m_{1}, n_{1}\right]$ is the total power emanating from the pixel $\left[m_{3}, n_{3}\right]$ and reaching the aperture of the microlens at $\left[m_{1}, n_{1}\right]$. We used the notation $\hat{I}$ to represent the power density in integral imaging associated with the points on $P_{3}$ and $P_{1}$ planes; $I$ is reserved for the power density between $P_{1}$ and $P_{2}$ planes; similar notation is used for $L$ and $\hat{L}$.

We place $P_{3}$ plane at $z_{3}$ and adjust the locations of $P_{1}$ and $P_{2}$ planes such that $P_{1}$ and $P_{3}$ planes become images of each other due to microlenses. Therefore, according to the lens magnification equation

$$
\frac{z_{1}-z_{3}}{z_{2}-z_{1}}=\left(\frac{S_{3}}{S_{2}}\right)^{1 / 2}
$$

where $z_{1}, z_{2}$ and $z_{3}$ are again the $z$-coordinates of the $P_{1}, P_{2}$ and $P_{3}$ planes, respectively, and $S_{2}$ is the area of the pixel $\left[m_{2}, n_{2}\right]$ which is the image of the pixel $\left[m_{3}, n_{3}\right]$ (Fig. 3).

Since the light power reaching to the microlens $\left[m_{1}, n_{1}\right]$ from an object point $\left(x_{0}, y_{0}, z_{0}\right)$ via the pixel $\left[m_{3}, n_{3}\right]$ on $P_{3}$ flows to the point $\left[m_{2}, n_{2}\right]$ on $P_{2}$ unchanged (lossless lenses), where $\left[m_{2}, n_{2}\right]$ is the image of $\left[m_{3}, n_{3}\right]$, using equation (5), we can write

$$
\begin{aligned}
\hat{I}_{d}\left[m_{3}, n_{3}, m_{1}, n_{1}\right] & ={ }^{\wedge} \mathrm{Id}[\mathrm{m} 3, \mathrm{n} 3, \mathrm{~m} 1, \mathrm{n} 1] \mathrm{S} 1 \mathrm{~S} 3 \\
& =\mathrm{P}[\mathrm{m} 3, \mathrm{n} 3, \mathrm{~m} 1, \mathrm{n} 1] \\
& =\operatorname{Id}[\mathrm{m} 1, \mathrm{n} 1, \mathrm{~m} 2, \mathrm{n} 2] \mathrm{S} 1 \mathrm{~S} 2
\end{aligned}
$$

Hence we can write

$$
\hat{I}_{\mathrm{d}}\left[m_{3}, n_{3}, m_{1}, n_{1}\right]=I_{\mathrm{d}}\left[m_{1}, n_{1}, m_{2}, n_{2}\right]\left(\frac{S_{2}}{S_{3}}\right)
$$

and this is consistent, as expected, with the lens magnification between the $P_{2}$ and $P_{3}$ planes. Together with equation (8), the two equations

$$
\begin{aligned}
\hat{L}_{\mathrm{d}}\left[m_{3}, n_{3}, m_{1}, n_{1}\right] & =\hat{I}_{\mathrm{d}}\left[m_{3}, n_{3}, m_{1}, n_{1}\right] \\
L_{\mathrm{d}}\left[m_{1}, n_{1}, m_{2}, n_{2}\right] & =I_{\mathrm{d}}\left[m_{1}, n_{1}, m_{2}, n_{2}\right]
\end{aligned}
$$

establish the desired equivalence either between $\left(P_{1}, P_{3}\right)$ plane pairs or $\left(P_{1}, P_{2}\right)$ plane pairs.

Therefore, the integral imaging can be represented as a discrete light field either between $P_{3}$ and $P_{1}$ or between $P_{1}$ and $P_{2}$ plane pairs, provided that the depth of focus of the microlenses is large enough to focus any point on the $3 \mathrm{D}$ object into a pixel on $P_{2}$ plane. ${ }^{7}$ Please also note that this equivalence is valid under the assumption that there is no cross-talk between the elemental images from different microlenses. In other words, we restrict the set of light frustum within a finite propagation angle behind each microlens such that overlaps are prevented. This can simply be achieved by partitioning the sensor array plane to non-overlapping regions such that each partition corresponds to the elemental image of a particular microlens and the leakage from a microlens to the elemental image of any other microlens is prevented. ${ }^{8}$

In order to relate the integral imaging to the light field representation in the continuous case, we need infinitely many infinitesimal microlenses on the infinite extent $P_{1}$, and infinitely many infinitesimal sensors on the infinite extent $P_{2}$ plane. Let us assume that the microlenses still possess the properties of an ideal lens even when their aperture sizes tend to zero. In this case, elimination of the cross-talk is practically impossible since each elemental image size will also be infinitesimally small. Hence, the integral imaging 
is not equivalent to the continuous light field representation.

In all previous discussions, we assumed that microlenses provide a sufficient angular resolution for our purposes. However, physically a microlens having an infinitesimal aperture size behaves like a point light source and diffracts the incoming ray in an equally weighted manner to all angles. Hence, its angular resolution will be zero. In other words, our ability to assign an arbitrary propagation angle distribution is lost. This practical issue is a direct consequence of the uncertainty principle which states that we cannot achieve infinite resolution in both time and frequency of a signal. ${ }^{9}$ Time-frequency representation corresponds to space-angle representation in our context. Therefore, the representation of the ray power densities with infinite resolution in both space and angle variables is impossible via the light field representation due to physical nature of light.

\section{CONCLUSION}

In conclusion, the integral imaging is equivalent to the discrete light field representation provided that the light rays are restricted within a finite propagation angle so that there is no cross-talk between the elemental images from different microlenses. The apertures of the microlenses and sensors correspond to pixels on the $P_{1}$ and $P_{2}$ planes, respectively, of the discrete light field representation. In the continuous case, the integral imaging is not equivalent to the light field representation since the elimination of the crosstalk between the elemental images becomes practically impossible. Furthermore, in this case, an infinitesimal microlens cannot keep its lens properties and does not provide infinite resolution in both space and angle since it behaves like a point light source and thus diffracts the incoming ray by equally distributing the incoming power in all directions. It is impossible to get infinite resolution in both space and angle due to physical nature of the light. These facts are the direct consequences of the uncertainty principle. At that point, it is necessary to incorporate the uncertainty principle into the formulation to obtain a more accurate model. Relating the light field representation to the integral imaging may result in important developments in integral imaging by linking the computer graphics approaches to it, and vice versa. The established link is also useful in understanding the limits of practical implementation of light fields.

\section{REFERENCES}

1 Adelson, E. H. and Bergen, J. R. In Computational Models of Visual Processing (Ed. M. Landy and J. A. Movshon), 1991, pp. 3-20 (MIT Press, Cambridge, MA).

2 Levoy, M. and Hanrahan, P. Light field rendering. SIGGRAPH, 1996, 96, 31-42.

3 Gershun, A. The light field. J. Math. Phys., 1939, 18, $55-151$.

4 Gortler, S. J., Grzeszczuk, R., Szeliski, R. and Cohen, M. F. The lumigraph. SIGGRAPH, 1996, 96, 43-54.

5 Lippmann, M. G. Epreuves reversible donnant la sensation du relief. J. Phys., 1908, 7, 821-825.

6 Martinez-Corral, M., Javidi, B., Martinez-Cuenca, R. and Saavedra, G. Formation of real, orthoscopic integral images by smart pixel mapping. Opt. Express, 2005, 13, 9175-9180.

7 Martinez-Cuenca, R., Saavedra, G., Martinez-Corral, M. and Javid, B. Enhanced depth of field integral imaging with sensor resolution constraints. Opt. Express, 2004, 12, 5237-5242.

8 Arai, J., Okano, F., Hoshino, H. and Yuyama, I. Gradient-index lens-array method based on real-time integral photography for three-dimensional images. Appl. Opt., 1998, 37, 2034-2045.

9 Hlawatsch, F. and Bartels, G. F. B. Linear and quadratic time-frequency signal representations. IEEE Signal Process. Mag., 1992, 9, 21-67. 